\title{
Prolonged respiratory failure due to pulmonary embolism in a young woman: a case report
}

\author{
Daniel Tuchscherer $^{1}$, Alexa Hollinger ${ }^{1 *} \mathbb{D}$, Jens Bremerich ${ }^{2}$ and Martin Siegemund ${ }^{1}$
}

\begin{abstract}
Background: The pathophysiology of pulmonary edema is generally considered to result from elevated pulmonary capillary hydrostatic pressure due to increased left atrial pressure in consequence of a failing left ventricle.

Case presentation: We present a case of pulmonary edema secondary to severe hypalbuminemia under excessive respiratory drive in a 29-year-old Caucasian woman in respiratory distress with detected bilateral central pulmonary embolism.

Conclusion: In conjunction with severe hypalbuminemia, even the negative intrathoracic pressure swings of respiratory distress may cause pulmonary edema. Detrimental consequences of non-invasive ventilation due to uncontrolled tidal volume and pressure swings need to be considered when treating patients in hypoxemic respiratory failure with low serum albumin.
\end{abstract}

Keywords: Hypalbuminemia, Pulmonary capillary hydrostatic pressure, Pulmonary edema, Respiratory drive, Starling's equation, Transpulmonary pressure

\section{Introduction}

The pathophysiology of pulmonary edema (PE) is generally considered a result of elevated pulmonary capillary hydrostatic pressure due to increased left atrial pressure in consequence of a failing left ventricle [1], and pulmonary embolism is normally ruled out in the presence of PE. According to Starling's equation, ${ }^{1}$ fluid flux across the pulmonary capillary wall is determined by the permeability of the vessel wall and net transmural driving pressure, which is a balance of hydrostatic forces that tend to move fluid out of the capillary and colloid osmotic forces that tend to keep fluid in the capillary. PE develops when the amount of fluid crossing the pulmonary capillary endothelium exceeds the capacity of lymph fluid efflux in pulmonary interstitial space [2].

* Correspondence: alexa.hollinger@usb.ch

${ }^{1}$ Department of Intensive Care, University Hospital Basel, Spitalstrasse 21, CH-4031 Basel, Switzerland

Full list of author information is available at the end of the article

\section{Case report}

A 29-year-old Caucasian woman in respiratory distress was referred for initiation of parenteral nutrition because of chronic malabsorption and malnutrition. She had a history of bariatric gastric bypass surgery 10 years prior with multiple revisions over the following decade due to persisting steatorrhea. Her nutritional history revealed a voluntarily reduced protein intake of $25 \mathrm{~g}$ per day. She smoked one pack of cigarettes per day (13 pack years). Alcohol and recreational drugs were negated. Family history was insignificant.

\section{Physical examination findings}

Her blood pressure (BP) was $132 / 58 \mathrm{mmHg}$, pulse rate was 120 beats per minute, respiratory rate 28 breaths per minute, and oxygen saturation $80 \%$ on room air. Auscultation revealed normal heart sounds and clear lungs. Lower extremity edema was noted and her right calf was tender on palpation. The rest of her clinical examination was unremarkable. 


\section{Diagnostic studies}

Routine laboratory analyses were normal except for hypalbuminemia $(8 \mathrm{~g} / \mathrm{L}$, normal range $35-52 \mathrm{~g} / \mathrm{L})$, elevated Bnatriuretic peptide (BNP; $844 \mathrm{ng} / \mathrm{L}$, normal value $<177$ $\mathrm{ng} / \mathrm{L}$ ), and C-reactive protein (CRP; $40 \mathrm{mg} / \mathrm{L}$, normal value $<10 \mathrm{mg} / \mathrm{L}$ ). Arterial blood gas analysis (BGA) confirmed hypoxemia: partial pressure of oxygen in arterial blood $\left(\mathrm{PaO}_{2}\right)$ of $8 \mathrm{kPa}$. A computed tomography (CT) scan of her chest revealed bilateral central pulmonary embolism and ground glass opacities (Fig. 1a) [3]. Echocardiography showed dilatation of her right ventricle with normal right ventricular function, while her left ventricle showed systolic D-shaping with normal systolic function [4]. Systolic pulmonary artery pressure (PAP) was estimated at 54 mmHg. Transpulmonary thermodilution and pulse contour cardiac output (Pulsion Medical Systems@) displayed a normal cardiac index $\left(4 \mathrm{~L} /\right.$ minute per $\left.\mathrm{m}^{2}\right)$, a slightly increased extravascular lung water index $(10 \mathrm{ml} / \mathrm{kg}$, normal range $3-7 \mathrm{ml} / \mathrm{kg}$ ), and a central venous pressure of 17 $\mathrm{mmHg}$. Because of worsening gas exchange over 3 days on non-invasive ventilation (NIV), a repeat CT scan was performed and profound ground glass opacities (Fig. 1b) were noted. Bronchoalveolar lavage (BAL) remained sterile.

\section{Diagnosis}

We diagnosed PE secondary to severe hypalbuminemia under excessive respiratory drive and treated her with thrombolysis, controlled mechanical ventilation, and albumin replacement.

\section{Discussion}

Pulmonary capillary hydrostatic pressure varies with blood flow in the pulmonary circulation and with distribution of pulmonary vascular resistance between precapillary and postcapillary vessels. A pulmonary embolism increases precapillary resistance and hence is usually not associated with increases in pulmonary capillary pressure and development of PE. However, this applies only for areas of the pulmonary vascular bed directly affected by pulmonary embolism [5]. Blood flow is redirected from occluded vessels to open vessels in which increased pressure may be transmitted to the capillary bed. Moreover, pulmonary vascular resistance is directly related to the degree of vascular obstruction and location of the embolus [6, 7].

On the other hand, increased respiratory drive in hypoxemic respiratory failure seems to play a more relevant role in the pathophysiology of PE in the presented case, in addition to hyperadrenergic state causing peripheral vasoconstriction and increase in venous return [8]. Increased venous return may further increase pulmonary blood flow thus contributing to edema. Cyclic changes of alveolar pressures during respiration affect pulmonary capillary hydrostatic pressure. The profound negative intrathoracic pressure swings during respiratory failure lead to a rise of pulmonary transcapillary pressure, the pathophysiologic basis of negative pressure PE [9]. During respiratory efforts, intravascular pressure measured in pulmonary intrathoracic vessels decreases, but to a lesser extent than the esophageal or pleural pressure, resulting in increased transmural pulmonary vascular pressures. Our patient showed high respiratory drive with respiratory rates around 30 breaths per minute and tidal volumes (TVs) of $12.5 \mathrm{ml} / \mathrm{kg}$ predicted body weight during the 72 hours of NIV. Increased respiratory drive with the resulting increase of transmural pulmonary capillary pressures probably represents the underlying pathomechanism of patient self-inflicted lung injury due to prolonged application of non-invasive mechanical ventilation in hypoxemic respiratory failure, which seems to be one of the reasons for NIV failure and poor
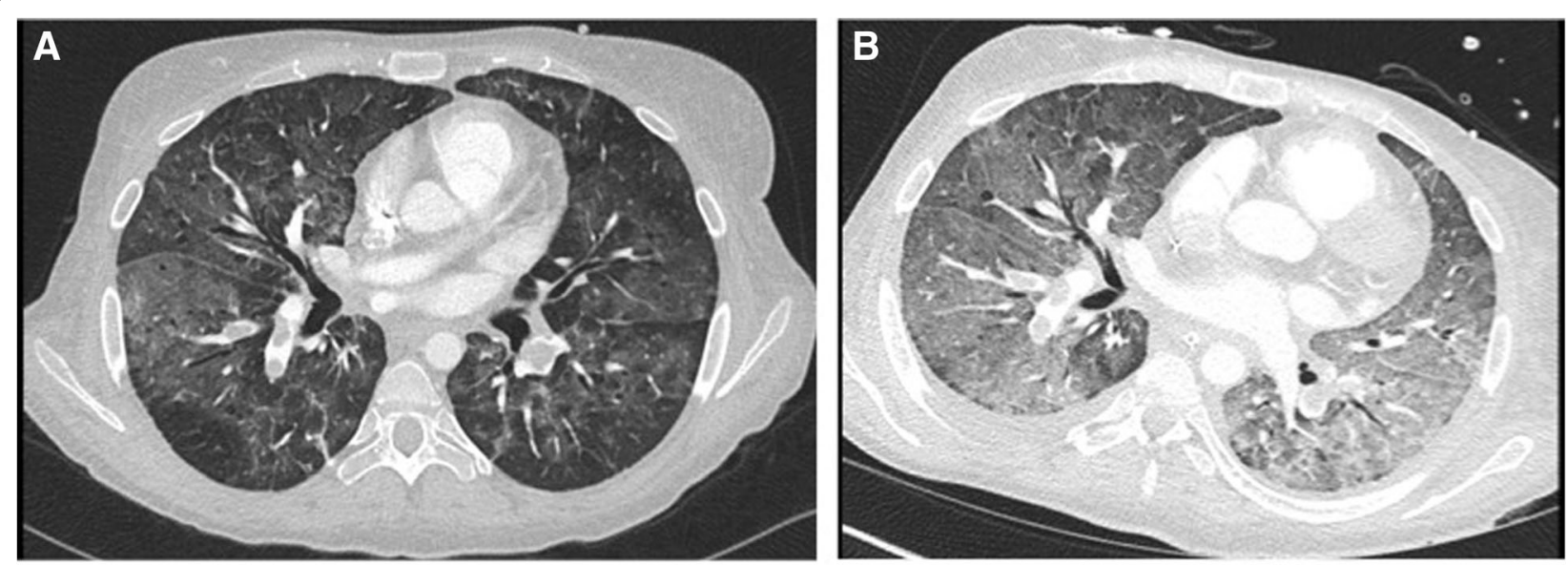

Fig. 1 a Computed tomography scan of chest on admission showing bilateral central pulmonary embolism and diffuse ground glass opacities. $\mathbf{b}$ Computed tomography scan of chest after 3 days of non-invasive mechanical ventilation showing profound ground glass opacities 
outcome of this patient group. As a consequence of negative pressure swings during experimentally increased respiratory resistance and during increased respiratory drive due to strenuous exercise, lung lymph flow was shown to double instantly. Increased transmural pulmonary vascular pressure in the context of an increased vascular permeability further increases the risk of PE through vascular leakage. Vascular permeability was shown to be increased in vascular injury inflicted by pulmonary embolism.

In addition to the described mechanisms favoring the development of PE, the counteracting determinant (that is, colloid osmotic pressure) was severely reduced in our patient, as serum albumin represents its main determinant. The effect of decreased albumin concentration on the development of PE is well known in experimental physiology. Low colloid osmotic pressure is considered a strong predictor in other lung diseases, such as acute respiratory distress syndrome (ARDS) [10]. The contribution of hypoalbuminemia to PE in clinical practice especially in the context of distinct negative pressure seems comprehensible, constituting hypalbuminemia as a risk factor for cardiogenic PE [11] and increased extravascular lung water in critically ill patients.

Finally, as the major determinant of interstitial fluid clearance is the pressure difference between the pulmonary interstitial space and the venous pressure at the junction of the thoracic duct and jugular vein, lymphatic flow may be significantly reduced in the setting of central pulmonary embolism and the associated rise in central venous pressure.

\section{Clinical course}

Our therapeutic approach targeted all of the components of Starling's equation. Systemic thrombolysis decreased pulmonary vascular resistance and restored right and left ventricular stroke volume balance. Lungprotective invasive mechanical ventilation minimized patient self-inflicted lung injury by reducing transpulmonary pressure swings and transmural pulmonary vascular pressure. Oncotic pressure was normalized by albumin administration and diuretic therapy. The alveolar PE slowly but continuously abated clinically and radiologically. After 12 days of invasive mechanical ventilation, our patient was discharged to the ward and transferred to a rehabilitation clinic 1 month after admission. On 6-month follow-up, she had fully recovered with normal static and dynamic lung volumes, mildly reduced carbon monoxide (CO) diffusion capacity, normal arterial BGA, as well as normal 6-minute-walking test and echocardiography.

\section{Conclusion}

Negative pressure PE is another well-described etiology of PE [7], possibly of importance in severe acute respiratory failure. In conjunction with severe hypalbuminemia, even the negative intrathoracic pressure swings of respiratory distress may cause PE. Part of the mechanisms underlying patient self-inflicted lung injury may be excessive respiratory drive with resulting increases in pulmonary transmural vascular pressures. Detrimental consequences of NIV due to uncontrolled TV and pressure swings [12] need to be considered when treating patients in hypoxemic respiratory failure with low serum albumin.

\section{Endnotes}

${ }^{1} \mathrm{Q}=\mathrm{Kf}\left[\left(\mathrm{P}_{\mathrm{c}}-\mathrm{P}_{\mathrm{i}}\right)-\mathrm{K}_{\pi}\left(\pi_{\mathrm{c}}-\pi_{\mathrm{i}}\right)\right]$ Starling's equation, where $\mathrm{Q}$ is the net fluid flow across the pulmonary capillary wall, $\mathrm{Kf}$ is the filtration coefficient (a measure of the permeability of the pulmonary capillary wall to fluid flux across it), $K_{\pi}$ is the oncotic reflection coefficient (a measure of the pulmonary capillary wall resistance to protein movement), $\mathrm{P}_{\mathrm{c}}$ is the pulmonary capillary hydrostatic pressure, $P_{i}$ is the pulmonary interstitial hydrostatic pressure, $\pi_{c}$ is the capillary oncotic pressure, and $\pi_{\mathrm{i}}$ is the interstitial oncotic pressure

\section{Abbreviations \\ ARDS: Acute respiratory distress syndrome; BAL: Bronchoalveolar lavage; BGA: Blood gas analysis; BNP: B-natriuretic peptide; BP: Blood pressure; CO: Carbon monoxide; CRP: C-reactive protein; CT: Computed tomography scan; NIV: Non-invasive ventilation; $\mathrm{PaO}_{2}$ : Partial pressure of oxygen in arterial blood; PAP: Pulmonary artery pressure; PE: Pulmonary edema; TV: Tidal volume}

\section{Acknowledgements \\ None.}

Accession number to microarray data Not applicable.

Clinical trial registration number

No registration was performed for this investigation.

Authors' contributions

$\mathrm{AH}, \mathrm{MS}$, and DT analyzed and interpreted the patient data. JB performed the examination of the CT image. DT wrote the manuscript. All authors have read and approved the final version (Version 1, 12.03.2019) of the manuscript.

\section{Funding}

No funding was received.

\section{Availability of data and materials}

The datasets used and analyzed during the current study available from the corresponding author on reasonable request. The clinical data are stored electronically in the intensive care clinical information system software (MetaVision, iMDsoft ${ }^{\oplus}$ ) provided in the intensive care units of the University Hospital Basel.

Ethics approval and consent to participate

The authors did not seek for approval from an ethics committee. The patient has given written consent to participate.

\section{Consent for publication}

The patient has given written informed consent for publication of her case report and any accompanying images. A copy of the written consent is available for review by the Editor-in-Chief of this journal. 


\section{Competing interests}

The authors declare that they have no competing interests.

\section{Author details}

'Department of Intensive Care, University Hospital Basel, Spitalstrasse 21, CH-4031 Basel, Switzerland. ${ }^{2}$ Department of Radiology and Nuclear Medicine, University Hospital Basel, Spitalstrasse 21, CH-4031 Basel, Switzerland.

Received: 20 March 2019 Accepted: 19 June 2019

Published online: 24 July 2019

\section{References}

1. Guyton AC, Lindsey AW. Effect of elevated left atrial pressure and decreased plasma protein concentration on the development of pulmonary edema. Circ Res. 1959:7(4):649-57.

2. Taylor AE. Capillary fluid filtration. Starling forces and lymph flow. Circ Res. 1981:49(3):557-75.

3. Thoma P, Rondelet B, Mélot C, Tack D, Naeije R, Gevenois PA. Acute Pulmonary Embolism: Relationships between Ground-Glass Opacification at Thin-Section CT and Hemodynamics in Pigs. Radiology. 2009;250(3):721-9.

4. Maclver DH, Clark AL. The Vital Role of the Right Ventricle in the Pathogenesis of Acute Pulmonary Edema. Am J Cardiol. 2015;115:992-1000.

5. Ehrhart C, Hall JE, Hofman WF. Vascular pressure effects on lung edema formation after glass bead embolism. J Appl Physiol. 1987;62(5):1989-96.

6. Elliott CG. Pulmonary physiology during pulmonary embolism. Chest. 1992; 101(4 Suppl):163S-71S

7. Nelson JR, Smith JR. The pathologic physiology of pulmonary embolism. A physiologic discussion of the vascular reactions following pulmonary arterial obstruction by emboli of varying size. Am Heart J. 1959;58:916-32.

8. Lemyze M, Mallat J. Understanding negative pressure pulmonary edema. Intensive Care Med. 2014;40(8):1140-3.

9. Arques S, Ambrosi P, Gelisse R, Luccioni R, Habib G. Hypoalbuminemia in elderly patients with acute diastolic heart failure. J Am Coll Cardiol. 2003; 42(4):712-6.

10. Arif SK, Verheij J, Groeneveld AB, Raijmakers PG. Hypoproteinemia as a marker of acute respiratory distress syndrome in critically ill patients with pulmonary edema. Intensive Care Med. 2002:28(3):310-7.

11. Bhattacharya M, Kallet RH, Ware LB, Matthay MA. Negative-Pressure Pulmonary Edema. Chest. 2016;150(4):927-33.

12. Brochard L, Slutsky A, Pesenti A. Mechanical Ventilation to Minimize Progression of Lung Injury in Acute Respiratory Failure. Am J Respir Crit Care Med. 2017:195(4):438-42.

\section{Publisher's Note}

Springer Nature remains neutral with regard to jurisdictional claims in published maps and institutional affiliations.

Ready to submit your research? Choose BMC and benefit from:

- fast, convenient online submission

- thorough peer review by experienced researchers in your field

- rapid publication on acceptance

- support for research data, including large and complex data types

- gold Open Access which fosters wider collaboration and increased citations

- maximum visibility for your research: over $100 \mathrm{M}$ website views per year

At $\mathrm{BMC}$, research is always in progress.

Learn more biomedcentral.com/submissions 